\title{
Proposal for a Method to Discriminate the Kind of Laundry that is Hung with Hanger
}

\author{
Takumi Saruhashi $^{\mathrm{a}}$, Ryunosuke Miyamoto ${ }^{\mathrm{b}}$, Shenglin $\mathrm{Mu}^{\mathrm{c}}$, Takaaki Akimoto ${ }^{\mathrm{a}}$, Yuhki Kitazono ${ }^{\mathrm{a}}$ \\ ${ }^{a}$ National Institute of Technology, Kitakyushu College,5-20-1 Shii, Kokuraminami-ku, Kitakyushu-city, Fukuoka \\ 802-0985, Japan \\ ${ }^{b}$ Molex Japan Co., Ltd., 1-5-4 Hukamihigashi, Yamati-city, Kanagawa 242-8585, Japan \\ ${ }^{\mathrm{c}}$ National Institute of Technology, Hiroshima College, 4272-1, Higashino, Osakikamijima-cho, Toyota-gun, Hiroshima \\ 725-0231, Japan \\ *Corresponding Author:kitazono@kct.ac.jp
}

\begin{abstract}
In this paper, we describe the system that removes laundry from hanger and folds in automatically. This system takes out the laundry each hanger from the hanger rack, and system detects whether the laundry is dry or not. If laundry is dry, hanger is removed from laundry and laundry is folded by the system. In generally, how to hang the laundry to the hanger is different depending on the kinds of laundry. In previous, we developed the mechanism that can remove the hanger from the laundry regardless kind of the laundry. However, the system couldn't discriminate the kind of the laundry. So, we propose how to discriminate the kind of laundry that is hung the hanger.
\end{abstract}

Keywords: folding the laundry, folding system, motor, Arduino, OpenCV.

\section{Introduction}

Housework is one of tough jobs. Not only that, we must do it every day. We can't live comfortably without doing housework. In the case of a single life, you have to do that as well as your work. On the other hand, if you have a large family, amount of housework becomes enormous. There are a great number of household tasks such as cleaning the room, washing the dishes, and doing the washing (1) (2). Previously, housework has been done manually. However, as a result of appearance of Dishwasher, Vacuum cleaner, and Washing machine, we can conveniently save trouble. Nevertheless, the machine which folds the laundry automatically has not prevailed among the ordinary homes. Even today, folding the laundry is a manual labor.

In 2015, the laundry folding robot: "Laundroid" was developed by Seven Dreamers Laboratories, Inc., Panasonic Corporation, and Daiwa House Industry Co.,Ltd. Though the robot has ability to fold many kind of clothes, it takes approximately 10 minutes to fold one T-shirt, and it doesn't consider whether the laundry has already dried or not. Due to that, user should check whether the laundry is dry when he takes the laundry in. Folding laundry in automatically has been studied by some researcher ${ }^{\text {(3) (4) }}$. However, the laundry is dried in the laundry dryer, so hanging the laundry to dry process is ignored in previous studies. When we take in account the percentage of households owning a laundry dryer, hanging out the laundry to dry is yet main means to dry the laundry ${ }^{(5)}{ }^{(6)}$. Addition to this, some clothes can't be dried in the laundry dryer because they shrink in the heat of the dryer. Also there are some attempts to keep a proper humidity of the room by hanging out the laundry to dry in the room to prevent the room from drying ${ }^{(7)(8)}$. If we hanged the laundry out, we have to detect whether the laundry is dried or not when we take the laundry in. After that, we have to remove the hanger from laundry to fold laundry if the laundry is dried. However, these processes of folding laundry are not much studied. From such background, we had developed the system to fold T-shirt in our previous study. The developed system could detect whether the laundry is dry or not. After detecting that the laundry is already dry, the system removes the hanger from the laundry and folds the laundry. It took time about 1 minute and 30 seconds to fold a laundry. The prototype of the system could fold only a T-shirt, later on, we improved the system. So, we had 


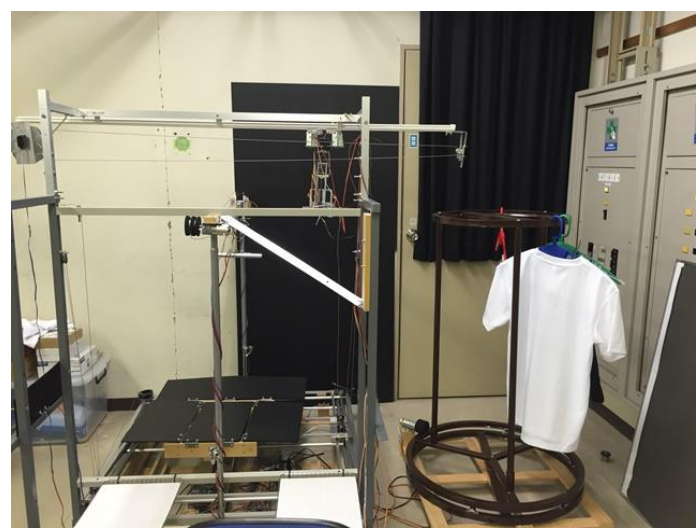

Fig. 1. Overall view of the system.

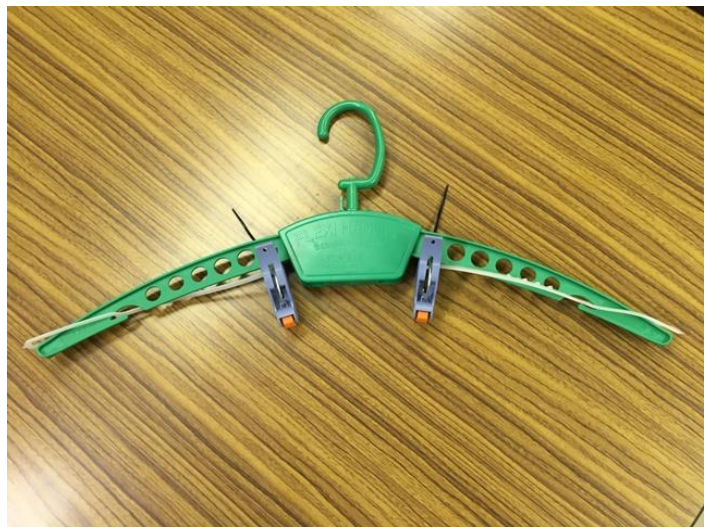

Fig. 2. The hanger and clothespins.

improved the removing hanger mechanism to deal with many kinds of laundry. The improved mechanism could remove hanger from laundry regardless kind of the laundry. However, the system needed information about the form of laundry to run improved mechanism properly. But the system didn't identify the kind of laundry. Due to that, the system couldn't remove the hanger automatically. We had to add the new function that identifies the kind of laundry.

So, we have developed the mechanism which discriminates the kind of the laundry. By introducing discrimination mechanism to the system, the system becomes able to identify the kind of the laundry. Also the removing hanger mechanism will be run properly and automatically by the system. From this, the system will be able to deal with many kind of the laundry.

\section{Construction of System}

\subsection{System overall view and the operating procedure}

The overall view of the system is shown in Fig.1. The rotary hanger rack part is shown in right side in the Fig.1.

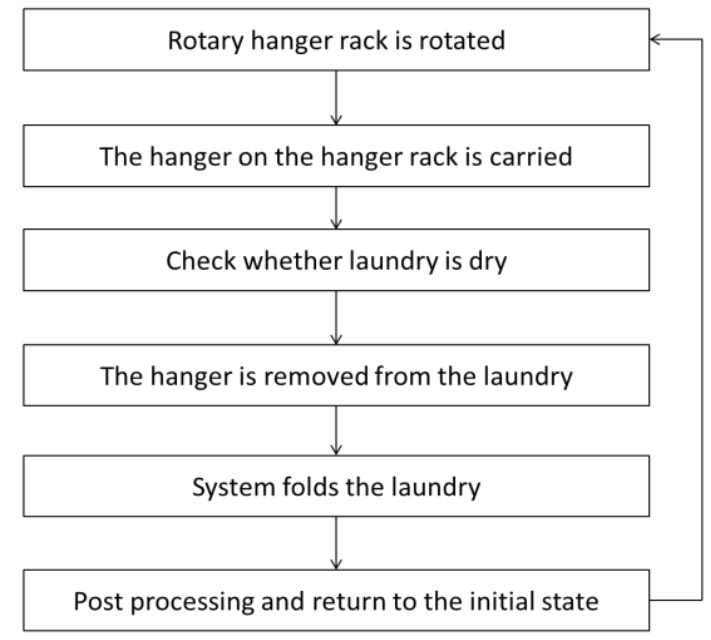

Fig. 3. System operation procedue.

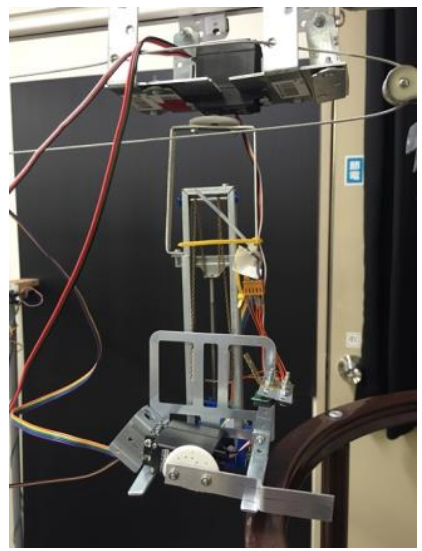

Fig. 4. Take out hanger mechanism.

The user hangs laundry on the rotary hanger rack. We use the clothes pin (NSA-37 by NAITO-KOGYOSHO, INC.) and hanger (DFQ011 by DAISAKU-SHOJI, INC) as shown in Fig.2 to hang laundry. We pinch the laundry to clothespins of the hanger. If the laundry has a sleeve such as a shirt, we hang the laundry to the hanger in normally. So, the sleeve is put through by the wings of the hanger. In Fig.2, the tip of the clothes pin is roller-like shape. The laundry that is pinched by the clothes pin can be taken easily by pulling the laundry or hanger. Also the hanger has special structure. Wings of the hanger are bent if the button of the hanger handle is pushed.

Folding laundry mechanism part is shown the left side in the Fig.1. This mechanism is the main part of the system. It detects dryness of laundry, removes hanger from laundry, and folds laundry.

The behavior of the system is shown in Fig.3. By using motors and operating the respective mechanism, these operations are realized. Also state of each mechanism is detected by sensors and motors, and they are controlled by 


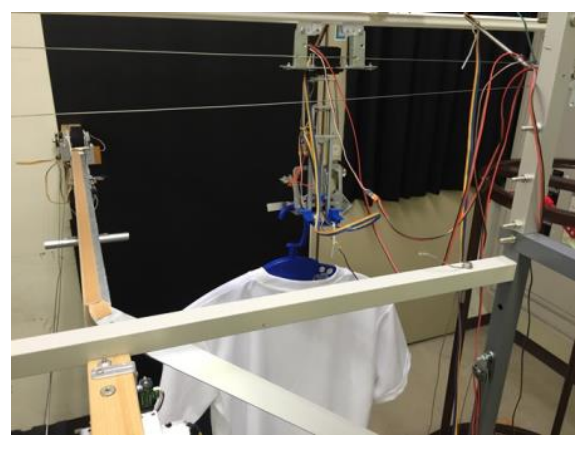

Fig. 5. The timing of discrimination.

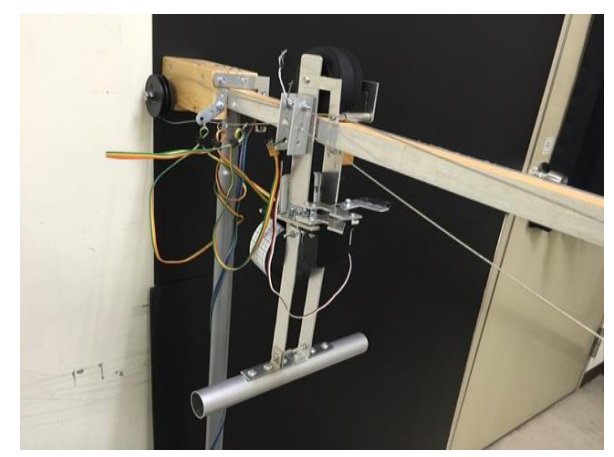

Fig. 6. The Hanger mobile device.

microcomputers. AC100V is used for the power supply of the system. It is converted to DC5V and DC12V by the power strip. The converted voltage is supplied to each mechanism.

\subsection{Mechanisms involved in the discrimination of the laundry}

The system will discriminate of the laundry when the laundry was carried from the rotary hanger rack to the folding laundry mechanism part. The take out hanger mechanism that carries the laundry is shown in Fig.4. The system carries laundry by the lift in mechanism. When the lift stops the movement in the middle of the rotary hanger rack and folding mechanism part, we will make the system discriminate of the laundry. The timing of discrimination is shown in Fig.5.

The removing hanger process is changed by the results of the laundry discrimination. The hanger mobile device that removes the hanger is shown in Fig.6. There are two methods to remove the hanger in this mechanism. One is pulling the hanger. This method is used when the laundry has no sleeve. The laundry is come off clothes pin by pulling hanger. Another, hanger mobile device pushes the button of hanger handle. Due to that, the wings of the hanger are bent and the hanger is taken through the collar of

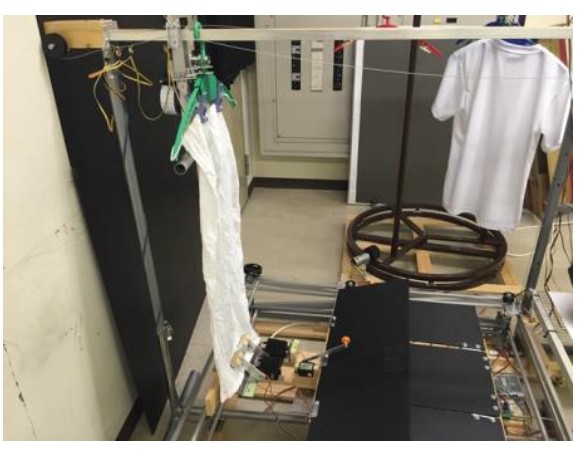

(a) The lower part of the laundry is fixed by arm of folding robot.

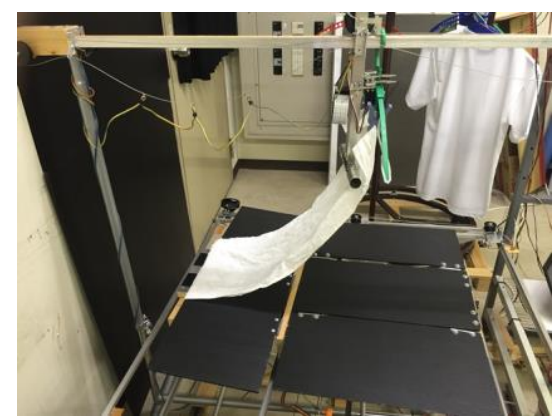

(b) The laundry is stretched by the system.

Fig. 7. Common process to remove the hanger.

the laundry. This way is used when the laundry has sleeve. These methods of removing the hanger are shown in Fig.7, 8 and 9. It is common process regardless of kind of the laundry to fix lower part of the laundry and stretch the laundry as shown Fig.7. Thereafter the mechanism has to run each method that differs according to the kind of the laundry as shown Fig.8 and 9. Therefore, the system must discriminate at least whether the laundry has sleeve or not.

\section{Discrimination of the Laundry}

For discrimination of the laundry, we used OpenCV for doing image processing. Also we use a camera for capturing the image of laundry. This time, we used background difference processing to discriminate whether the laundry has sleeve or not. The discrimination procedures are summarized as follows.

First, takes a picture as a background. After that, takes a picture that appeared moving object therein. This time, the object is the laundry that was hung the hanger. Two images are converted to grayscale in order to simplification of the calculation between each picture. Thereafter, we calculate the absolute value of the difference between the pixel values of two grayscale images. Later on, based on the result of calculation, the new grayscale image is 


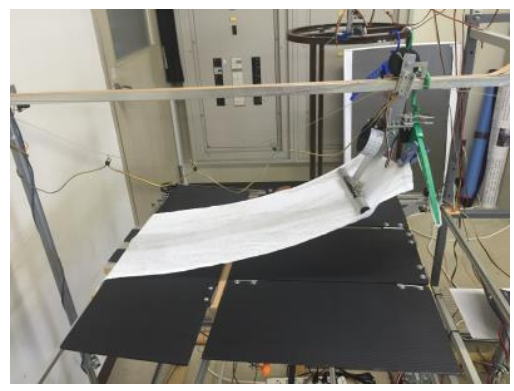

(a) The towel is stretched.

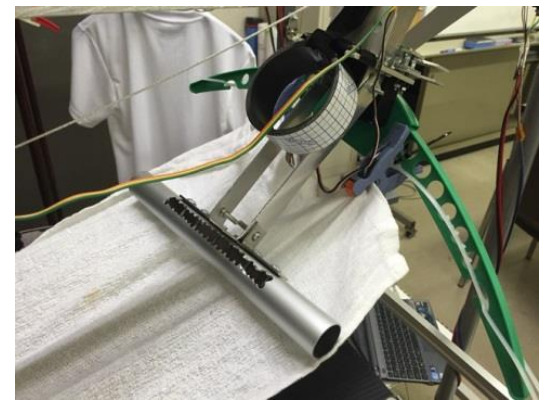

(b) The clothes pins are come off the towel.

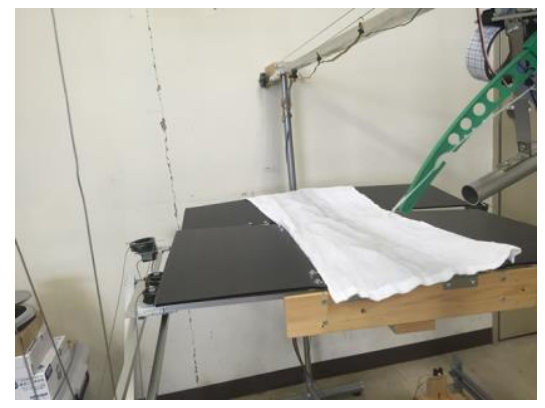

(c) The hanger is removed from towel.

Fig. 8. In the case of the laundry has no sleeve.

generated. By this process, in the generated image, the portion that is not changed in the two grayscale images becomes dark, and changed portion becomes bright. Based on it, the binary image is generated. In the binary image, background is represented by black and the object is represented by white. For noise rejection, the pixels of white color in the binary image are magnified and result after magnified is demagnified. Finally, we calculate white area of the particular area in the binary image for discrimination laundry. We can discriminate laundry to change calculation area in the image. If we calculate white area around the hanger wings in the binary image, we can discriminate whether the laundry has sleeve or not from calculation value. So, the calculation value becomes large if the laundry has sleeve.

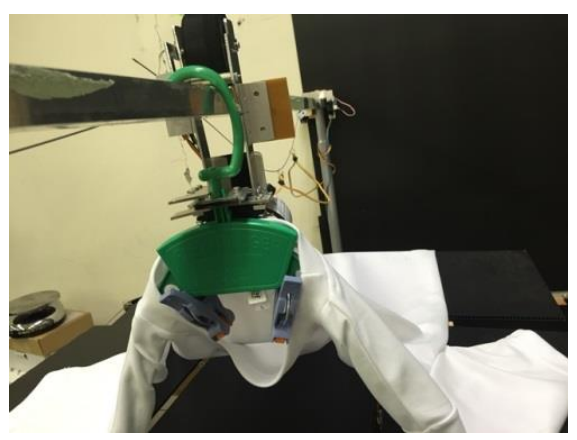

(a) The hanger handle button is pushed.

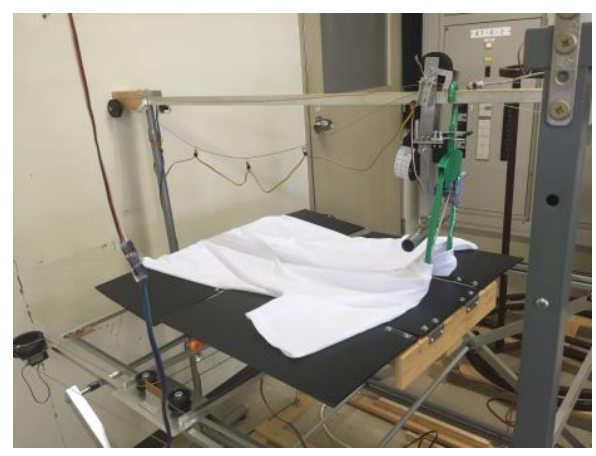

(b) The hanger is removed from T-shirt.

Fig. 9. In the case of the laundry has sleeve.

\section{Experiment}

We checked the performance of the discrimination laundry mechanism. We took images; background, T-shirt, towel. About the background, we used plastic cardboards to prevent assimilation of laundry and background. These images are shown in Fig.10. We performed the image processing and discriminated the laundry whether it has sleeve or not.

\section{Result of Experiment}

As a result of experiment, the binary images as shown in Fig.11 are generated. We calculate white area of the particular part in Fig.11. The part that was calculated is around the end of the left wing of the hanger in Fig.10(a),(b). By results of calculation, the significant difference is obtained from Fig.10(a) and (b). As a result, the system could discriminate the laundry whether it has sleeve or not. But, as can be seen from Fig.10 and 11, the hanger does not appear in Fig.11. So, the hanger was considered the same as the background. We tried to same experiment using black clothe. As a result of that, the binary image is generated, but the image reflects nothing. 


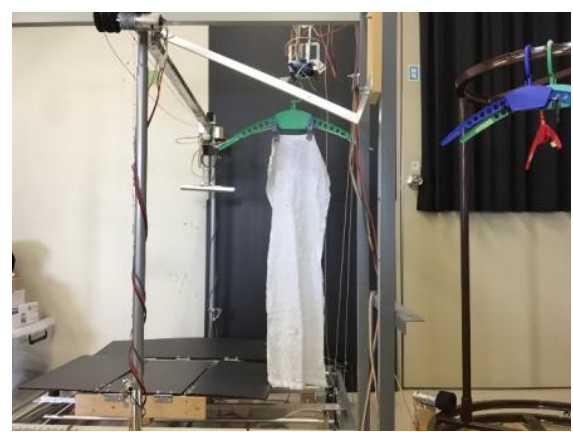

(a) Towel.

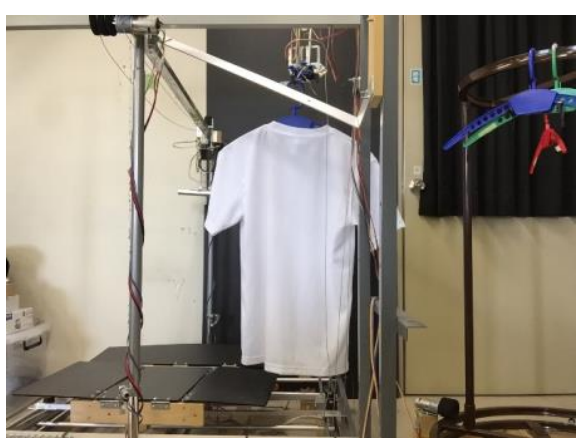

(b) T-shirt.

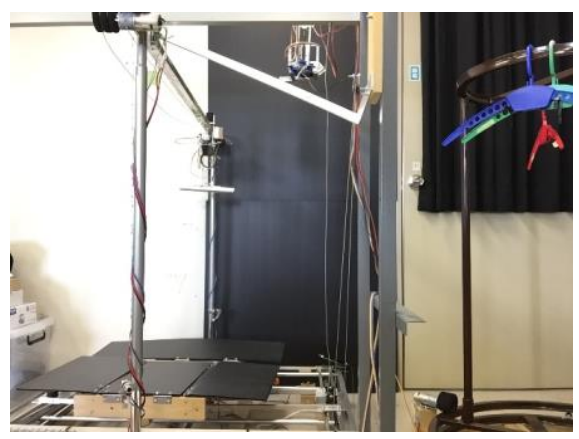

(c) Background.

Fig. 10. Experiment of discrimination of laundry.

So, the laundry wasn't detected if the background color and clothe color are same.

\section{Conclusions}

As a method of laundry discrimination, we understood that background difference processing is effectiveness by the experiment. But we must prevent assimilation of laundry and background.

If we prepare white background in the reverse direction in the experiment and take picture in the reverse, the laundry may be discriminated regardless of the color of laundry. So, if we perform the image processing about

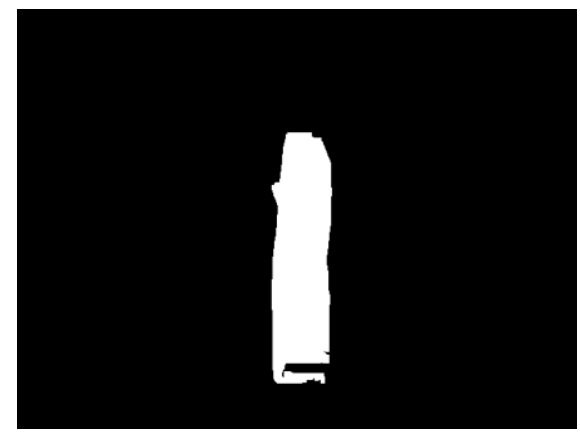

(a) Result of image processing of Fig.10(a).

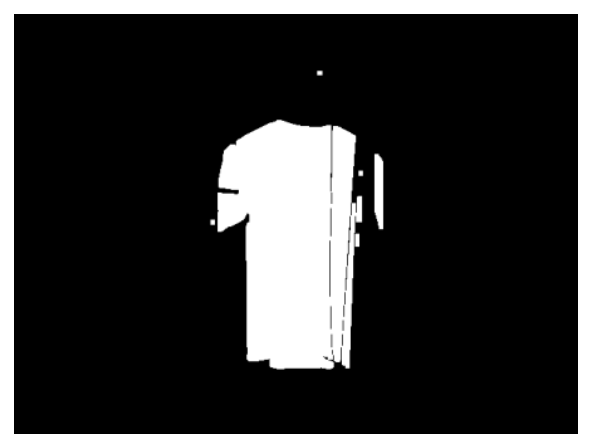

(b) Result of image processing of Fig.10(b)

Fig. 11. Result of image processing.

black and white background and calculate the logical OR of them, the system will discriminate the laundry. We will implement of these.

\section{References}

(1) Kabata Shinichiro : "Technological Progress of Washing Machine", Journal of Japan Society of Mechanical Engineers, Vol. 115, No. 1120, pp. 146-147, 2012

(2) Nishiwaki S. : "Tecqnology Trend of Washing Machine", Journal of Japan Society of Mechanical Engineers, Vol. 115, No. 1120, pp. 146-147, 2012

(3) Hamajima Kyoko, Kakikura Masayoshi : "Planning Strategy for Task of Utangling Laundry - Isolation of Clothes from Washed Mass -", JRM, Vol. 10, No. 3, pp. 244-251, 1998

(4) Kaneko Manabu, Kakikura Masayoshi : "Study on Handling Clothes (Task Planning of Deformation for Unfolding Laundry)” JRM, Vol. 15, No. 4, pp. 406-415, 2003

(5) Fukuda Eiko : "Survey of Home Washing", The journal of Wayo Women's University, Vol. 43, pp. 85-98, 2003

(6) Koitabashi Emiko, Okita Fumiko : "The Actual 
Conditions of Washing Work/Working Space and Problems to Be Solved : From an Investigation Carried Out in 2000 and in 2008", Memoirs of the Japan Women's University. Faculty of Home Economics, Vol. 57, pp. 95-106, 2010

(7) Kazue Shoushi, Sato Manami : “41159 Study on control methods of relative humidity in a room to use desorption from finishing material and clothes : Part 1 An evaluation of hygrothermal distribution by actual measurement", Summaries of technical papers of Annual Meeting Architectural Institute of Japan. D-2, Environmental engineering II, Heat, moisture, thermal comfort, natural energy, air flow, ventilation, smoke exhaustion, computational fluid dynamics, indoor air quality heating, cooling and air-conditioning heat and cold sources, piping systems application of building services 2008, pp. 317-318, 2008

(8) Kazue Shoushi, Sato Manami : “41160 Study on control methods of relative humidity in a room to use desorption from finishing material and clothes : Part 2 An numerical analysis of hygrothermal distribution", Summaries of technical papers of Annual Meeting Architectural Institute of Japan. D-2, Environmental engineering II, Heat, moisture, thermal comfort, natural energy, air flow, ventilation, smoke exhaustion, computational fluid dynamics, indoor air quality heating, cooling and air-conditioning heat and cold sources, piping systems application of building services 2008, pp. 319-320, 2008 